liquid that rises higher than the other stands steadily at a convenient marked point, when the air-pump is kept vigorously going, with the lower air-pump stopcock closed. This marked point may be perhaps a few centimetres below the india-rubber collar, so as to allow the liquid surface of it to be conveniently seen through a wide glass cylinder containing hot or cold water around it, applied to fulfil the thermal conditions referred to in Operation No. 6. In these present circumstances the vapour pressure is practically equal throughout the upper bent tube and the portions of the glass tubes between its ends and the liquid surfaces in the two glass tubes. Hence the more volatile of the two liquids is kept cool at its surface by rapid evaporation, and the less volatile liquid is kept warm by rapid condensation of vapour into it, so that, by flow of vapour through the bent tube, the difference of temperatures required to equalise the vapour pressures is very nearly maintained.

Operation No 5.-Close the upper three stopcocks, both air-pump stopcocks being already closed, and the two lowest metal stopcocks open. Leave the apparatus to itself until the temperatures become equalised. The difference of levels of the liquids in the two glass tubes, with proper corrections for their densities and for the difference, if any, of levels of the liquid surfaces in the two bottles, measures accurately the difference of vapour pressure over them, at the temperature to which they become equalised.

Operation No.6.-Open the upper air-pump stopcock, work the air-pump and open the stopcock over the top of one of the two liquids for a minute or two and close it again. Do the same for the other liquid. Allow temperatures to be equalised to what they were at the end of Op. 5. If any air or other foreign volatile substance ${ }^{1}$ has escaped from either liquid along with its proper vapour, its level will be seen higher than it was at the end of Op. 5. The present operation (No.6) must be continued long enough to distil out of either, or both liquids, any such foreign ingredients if, when originally introduced, any such impurity was contained.

Operation No. 7.--By proper thermal appliances, indicated by the dotted lines in the diagram, and the lamp under the upper bent metal tube (inserted merely as an indication that somehow the metal tube is to be always slightly warmer than the warmer of the two liquid surfaces, in order that there may be no condensation of vapour in it), bring the upper surfaces of the liquids to any other temperature, or to two different temperatures. The difference of levels of the liquids in the two tubes, with proper correction for the densities of the two liquids at their actual temperatures in different parts of their columns, gives the difference of vapour pressures for the actual temperatures of the two liquids at their upper surfaces.

Operation No. 8.-To facilitate and approximately determine the hydrostatic correction for specific gravities at the actual temperatures of the two liquids, open wide the stopcocks above the tops of the two glass tubes, and let a little air run back from the air-pump, by very cautiously and slightly opening our upper air-pump stopcock, and closing it again before the lower of the two liquid surfaces reaches the lower end of its glass tube. After that, by cautiously opening and closing our lower air-pump stopcock, let in a little air to the bottles until the mean level of the liquids in the two columns rises to nearly the same level as it had in the measured positions of $\mathrm{Op} .5$ or $\mathrm{Op} .6$. In the present circumstances, air in the upper bent metal tube resists diffusion of vapour through it sufficiently to prevent any important difference of temperatures from being produced by evaporation and condensation at the two liquid surfaces, and there is

1 See Ostwald, " Physico-Chemical Measurements,' translated by Walker Macmllian, $189^{2}$ ), last paragraph, page $\mathrm{Ir}$

NO. I 42 I, VOL. 55$]$ practically perfect hydrostatic equilibrium of equal liquid pressures at the tops of the two columns.

The vapour pressure of water is accurately known through a very wide range of temperature from Reg. nault's experiments ; hence, if pure water be taken for one of our two liquids, the mode of experiment described above determines the vapour pressure of the other liquid.

The apparatus may be kept day after day with the same liquids in it (all the stopcocks to be closed, except when it is not in use for observations); and thus, the observations for difference of vapour pressures may be repeated day after day ; or a long series of observations may very easily be made to determine vapour pressures at different temperatures. Always before commencing observations, Operation 6 must be repeated to remove air or other impurity, if any air has leaked in, or if air or other foreign volatile impurity has escaped from dissolution in either liquid into the vapour space above it. KELVIN.

\section{RELATIVE TEMPERATURES IN GEISSLER} TUBES.

I $\mathrm{N}$ the Physical Institute of the Berlin University, $\mathrm{Mr}$ R. W. Wood has been making a series of experiments, most interesting to students of astrophysics, with the object of investigating the relative temperatures at different parts of the discharge in a Geissler tube, with special reference to the stratification phenomena. Wiedemann and Hittorf, and also the theoretical calculations of Warburg, have shown that the temperature of the gas in the positive part of the discharge lies far below red heat, while that of the negative light, according to Hittorf, is at least below the melting-point of platinum. These observations are for the most part corroborated by the experiments of Mr. Wood, who has investigated in this case a fixed part of the discharge in an atmosphere of nitrogen under varying pressures and currents of different strengths. The results obtained by employing hydrogen instead of nitrogen established the fact that, under sinilar conditions of pressure and strength of current, the heating was only about i I per cent. of that found in the former case. It was found difficult, however, to keep a steady current with this gas.

Perhaps more interesting are the results which he has been able to procure by determining the relative temperatures of the different parts of the space between the anode and kathode. For this he has designed a neat and very simple means, by which the positions of the bolometer inside the vacuum tube might be varied at will without impairing in the least degree the vacuum. The description of this apparatus will be found in the article in which the results of his observations have been published (Physical Reviez, NovemberDecember 1896, xxi.). We may, however, mention that the bolometer wire--that is, the wire which was placed in the different positions between the two poles of the Geissler tube to indicate the varying temperatures of the different parts of the discharge-was here composed of platino-iridium, and bent in the form of a loop. Its exact position could at any moment be read off from a vertical scale. It was thus found possible to make a complete map of the temperature changes inside the vacuum tube.

In the unstratified anode light the temperature was sometimes constant for the greater part of the column, rising to a maximum near the middle, and falling off as the dark space was approached. The maximum was always found when the light was on the point of stratifying, and sometimes at higher pressures. The exact conditions, however, could not be determined; but the extent of the anode light played an important part. 
On nearing the dark space, a decrease of temperature was always observed. The temperature was found to drop very suddenly on leaving the anode light, reaching a minimum near the middle of the dark space ; a rapid rise to maximum occurred as the blue negative light was entered. With a pressure sufficiently reduced to cause the appearance of stratifications in the anode light, the maximum was always to be found in the middle of the column, the temperature rising as the anode was left behind, and falling after the middle of the column was passed. In addition, "there is a periodic rise and fall, the light discs being warmer than the dark spaces between them, although one often finds a point where there is no change of temperature on passing from a light space to a dark." This last-mentioned fact is explained on the ground that the increase in the steepness of the curve as the maximum is approached, masks the comparatively small decrease due to the passage from the light to the dark interspaces.

One of the many diagrams he reproduces, shows the temperature fluctuations in the stratified discharge at a low pressure of $\mathrm{O}^{\circ} \mathrm{I} \mathrm{mm}$. The ordinates increase for a rise in temperature, and the abscissæe are longer the further the bolometer wire is away from the anode. The horizon of the diagram is taken as the temperature of the room, which in this case was $25^{\circ}$. Comparing the curve giving the fluctuations of the bolometer wire placed at points of different intensities throughout the tube (the latter being drawn parallel to the abscissæ), many points of interest may be at once seen. Commencing at the anode, the curve on the whole is fairly horizontal, but rises wave fashion at every increase of luminosity in the tube, dropping more or less suddenly as a dark space is entered. As the kathode is approached, the bolometer wire enters the large dark space; the curve falls somewhat abruptly down for some distance, rising again rapidly as the kathode is approached.

It may be stated that the maximum in the anode light is less predominant here than it is at higher pressures, owing to the smaller changes of temperature.

The periodic change in the stratified anode light was made the subject of a detailed investigation, more points of reference being taken. These results were also plotted in the diagram just mentioned (larger scale). "The temperature is steady for a certain distance, then rises gradually to a maximum, situated in the brightest part of the disc, turns and drops suddenly as we pass out of the sharply defined edge of the disc. The difference of temperature between the light and dark spaces varies from $0^{\circ} 5$ to about $\mathrm{I}^{\circ} 5$, depending on the degree of exhaustion and current strength."

Assuming the electrical energy is wholly connected with heat, the temperature curves indicate for

Positive light ... Medium potential fall .. Medium temperature

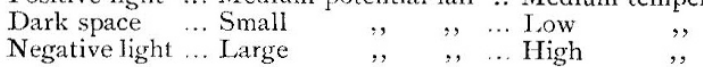

a result which, as Mr. Wood says, agrees with what is already known.

Two incidental points of interest mentioned refer to the behaviour of the strata. The movement of the bolometer loop from one stratum to another appears, at some pressures, to draw the stratum through which it is passing into the one immediately below it, the two dissolving into one, and the place left thus vacant being filled up by a new stratum springing off the anode. The NO. I 421 , VOL. 55] edges of these strata further act as if they "had an elastic skin or a sort of surface tension, bending in as the wire pushes against them, and finally snapping back to their original positions, leaving the wire well within the luminous disc." Mr. Wood, commenting on the results, considers that the curves obtained with the movable bolometer indicate with considerable accuracy the relative temperatures in the different parts of the discharge.

\section{THE TOMB OF LOUIS PASTEUR.}

$A \mathrm{~N}$ account of the impressive ceremony with which the remains of Pasteur were laid in their last resting-place at the Pasteur Institute was given in these columns on December $3 \mathrm{I}, \mathrm{I} 896$. We are indebted to the Lancet of January 9 for the following full description of the tomb, and for the accompanying illustration, which is reproduced from a fine picture of the mausoleum of the great French investigator.

The mausoleum is built at the end of a long corridor in the Institute, and is shut off by magnificent gates of wrought iron. 